\title{
Diels-Alder cycloaddition and RAFT chain end functionality: an elegant route to fullerene end-capped polymers with control over molecular mass and architecture
}

\author{
Anna Isakova, ${ }^{1}$ Christian Burton, ${ }^{2}$ Daniel J. Nowakowski, ${ }^{3}$ Paul D. Topham ${ }^{2 *}$ \\ 1. School of Chemical Engineering \& Advanced Materials, Merz Court, Newcastle \\ University, Newcastle upon Tyne, NE1 7RU, UK. \\ 2. Aston Institute of Materials Research, School of Engineering and Applied Science, \\ Aston University, Birmingham, B4 7ET, UK. \\ 3. Bioenergy Research Group, European Bioenergy Research Institute, Aston \\ University, Birmingham, B4 7ET, UK.
}

\begin{abstract}
Fullerene C60 functionalised polymers (FFPs) have found numerous applications from photovoltaic devices to materials for photodynamic therapy. Polymer end-capping is one way to fabricate FFPs since it provides enhanced control over the macromolecular architecture and composition. This paper reports, for the first time, a facile, metal catalyst-free approach to FFPs where polymers, generated by reversible-addition fragmentation chain transfer (RAFT) polymerisation, were coupled to a fullerene derivative through chain-end functionality, provided by the chain transfer agent without further modification. Two routes to a fullerene derivative were compared - based on Prato reaction and Diels-Alder cycloaddition. The Diels-Alder route exclusively yielded the mono-addition product, whereas the Prato route resulted in a mixture of monoand diadducts which required further separation. This elegant combination of welldefined RAFT polymerisation and precise Diels-Alder addition allowed one to obtain
\end{abstract}


fullerene end-capped polymers within a wide range of molecular masses (from 5,000 to $50,000 \mathrm{~g} / \mathrm{mol})$.

\section{INTRODUCTION}

For more than 30 years since the discovery of the fullerene molecule,${ }^{1}$ fullerene-functionalised materials have been attracting significant research interest due to the many fascinating properties of fullerenes such as photoconductivity, ${ }^{2-4}$ photorefractivity, ${ }^{5}$ high electron affinity (particularly useful for photovoltaic devices),${ }^{6-9}$ antioxidant properties ${ }^{10,11}$ and their ability to generate singlet oxygen. ${ }^{12}$ Combined with the tunability, flexibility, plasticity, solubility, processability, biocompatibility or biodegradability of polymers, these properties make fullerene-functionalised polymers (FFPs) an incredibly diverse group of materials with an extensive range of existing and potential applications.

Various approaches have been used to functionalise a polymer with a C60 molecule, ${ }^{13}$ the most common being: (i) grafting $\mathrm{C} 60$ onto the polymer through pendent groups ${ }^{14-16}$ and (ii) attaching a fullerene derivative on the end of the polymer chain (so called 'end-capping'). The former approach, although providing a significantly higher loading of fullerene molecules per polymer chain, allows little control over precise grafting density and thus over the final fullerene content. On the other hand, the latter approach is limited to one C60 molecule per chain (or two if the functionalisation occurs at both polymer termini) but gives a better control over the molecular composition and structure of the final polymer. Generally, fullerene functionalisation requires elaborate multi-step chemistry procedures due to the poor solubility of fullerenes in low-boiling point solvents, its UV-light and air sensitivity, affinity to dimerisation, formation of unpredictable by-products, poor control over the addition stereochemistry and difficulties with purification and characterisation due to large molecular volume and mass. ${ }^{17-19}$

Most of the chain end-capping reactions employ $[6,6]$-phenyl- $\mathrm{C}_{61}$-butyric acid (PCBA) which is coupled to hydroxy-functionalised polymers via Steglich esterification. ${ }^{6,15,20,21}$ Although 
efficient, the procedure normally requires additional steps of demethylation of commercially available [6,6]-phenyl- $\mathrm{C}_{61}$-butyric acid methyl ester (PCBM), and functionalisation of the polymers with the appropriate chain end. To reduce the number of steps, one can exploit controlled polymerisation methods, such as reversible addition-fragmentation chain transfer (RAFT) or atom transfer radical polymerisation (ATRP) to produce polymers with designated chain end groups in addition to having control over molecular mass and dispersity. ${ }^{22,23} \mathrm{~A}$ notable example of addition of fullerene to a chain end involved azide-alkyne click chemistry, where alkyne-functionalised fullerene was reacted with a polymer bearing a terminal azide group. ${ }^{24,25}$ The polymers were generated via ATRP and thus boasted low dispersity and controlled molecular mass, however, the overall functionalisation procedure had at least four steps: (i) polymerisation; (ii) conversion of the brominated polymer end (intrinsically generated during ATRP) into an azide group; (iii) synthesis of alkyne-functionalised fullerene and (iv) click coupling. To reduce the number of steps, it is possible to directly react the polymer- $\mathrm{Br}$ with C60, using a copper (or Grignard) catalyst. ${ }^{26-28}$ This approach poses two major problems as it typically gives rise to a number of by-products as a result of multiple polymer attachments onto one fullerene molecule ${ }^{17,29-31}$ and any residual metal can detrimentally affect the performance of the material (e.g., within organic electronic devices) or significantly increase its toxicity. Ideally, metal-catalysed reactions should be avoided to negate the requirement of extensive purification.

In this report, an elegant and simple method is demonstrated to cap a polymer chain end with a single C60 molecule without the use of metal catalyst or extensive purification. As a demonstration of this approach, catechol-inspired metal-binding polymers with controlled predetermined molecular mass have been generated by RAFT, which afforded chain end functionality necessary for simple attachment of the polymer to a well-reported Diels-Alder fullerene derivative. This method generated fullerene-functionalised polymers in a manner that allowed control over the mono-addition of a fullerene to the polymer whilst controlling the molecular mass of the whole macromolecule. In contrast, a comparative method using 
fullerene derivatives generated by the Prato reaction resulted in a mixture of mono- and diadducts which required further separation and purification of the product, demonstrating the advantages of the Diels-Alder route for exquisite control over the synthesis of polymerfullerene coupled materials. Moreover, as our approach employs a RAFT-generated macromolecule, this method is amenable to a wide range of monomer building blocks with ultimate control over the final molecular mass, composition and architecture of the desired polymer construct.

\section{EXPERIMENTAL}

\subsection{Materials}

Poly(3,4-dimethoxystyrene) (PDMS, $M_{\mathrm{n}} \sim 5,000,10,000$ and 50,000 $\mathrm{g} / \mathrm{mol}$ ) was synthesised according to our previous report ${ }^{32}$. Fullerene C60 (Solenne BV), 4-hydroxybenzaldehyde (98\%), sarcosine (98\%), 2-(trimethylsilyloxy)-1,3-butadiene (98\%), diisobutylaluminium hydride (DIBAL-H, 1.0 M in cyclohexane), N,N'-dicyclohexylcarbodiimide (DCC, 99\%), 4(dimethylamino)pyridine (DMAP, ReagentPlus ${ }^{\circledR}, \geq 99 \%$ ), triethylamine (TEA, $\geq 99 \%$ ), carbon disulfide (anhydrous, $\geq 99 \%$ ), 1,2-dichlorobenzene (anhydrous, all Sigma Aldrich), dichloromethane (DCM), toluene, hexane and methanol (all Fisher Scientific, Laboratory grade) were used as received.

\subsection{Synthesis of poly(3,4-dimethoxystyrene)}

Poly(3,4-dimethoxystyrene) (PDMS, $M_{\mathrm{n}} \sim 5,000,10,000$ and 50,000 $\mathrm{g} / \mathrm{mol}$ ) was synthesised according to our previous report with modifications. ${ }^{32}$ The following example describes the polymerisation of DMS with a target degree of polymerisation, Dp of $30\left(M_{n} \sim 5,000 \mathrm{~g} / \mathrm{mol}\right)$; this is representative of all DMS polymerisations undertaken in this work. AA $50 \mathrm{ml}$ round bottom flask equipped with a magnetic follower bar was charged with a mixture of DMS $(6 \mathrm{~g}, 36.54$ mmol), AIBN (10 mg, $0.06 \mathrm{mmol}$ ), DDMAT (444 mg, $1.22 \mathrm{mmol}$ ), and THF (12 mL). The flask was sealed with a rubber septum, and the solution was stirred and purged with nitrogen for 15 min, following which the flask was placed in an oil bath at $60^{\circ} \mathrm{C}$. After 24 hour stirring, the 
polymerisation was cooled rapidly to $0^{\circ} \mathrm{C}$ to allow immediate termination. $30 \mathrm{~mL}$ of THF was added, and the resulting solution was precipitated in $500 \mathrm{ml}$ of hexane. The precipitate was collected by filtration, washed with hexane several times, and dried in vacuo to obtain a pale yellow powder.

\subsection{Synthesis of fullerenol precursor (FULP-OH) (Route 1)}

A mixture of C60 (200 mg), 4-hydroxybenzaldehyde (40 mg) and sarcosine $(1.0 \mathrm{~g})$ in 1,2dichlorobenzene $(60 \mathrm{~mL})$ was stirred overnight at $150^{\circ} \mathrm{C}$ under a nitrogen atmosphere. The solvent was removed by rotary evaporation under reduced pressure. The crude product was purified by silica gel column chromatography with toluene to toluene:methanol (1 to $1: 1)$ to afford a brown solid (170 mg, yield 70\%). ${ }^{1} \mathrm{H}$ NMR ( $\delta$, benzene- $\left.\mathrm{d}_{6}+\mathrm{MeOD}\right): 7.6(2 \mathrm{H}, \mathrm{d}), 6.8$ $(2 \mathrm{H}, \mathrm{d}), 4.9(1 \mathrm{H}, \mathrm{d}), 4.65(1 \mathrm{H}, \mathrm{s}), 4.25(1 \mathrm{H}, \mathrm{d}), 2.4(3 \mathrm{H}, \mathrm{s})$.

\subsection{Synthesis of fullerenol precursor (FUL-OH) (Route 2)}

Procedure adapted from An et al. ${ }^{12}$ with modifications. $1.5 \mathrm{~mL}$ of 2-[(trimethylsilyl)oxy]-1,3butadiene was slowly added via a syringe pump to a refluxing solution of $1.0 \mathrm{~g}(1.39 \mathrm{mmol})$ of fullerene $\mathrm{C} 60$ in $400 \mathrm{~mL}$ of dry toluene under inert atmosphere. After addition, reflux was continued for $24 \mathrm{~h}$ and the reaction mixture was cooled to room temperature. The solvent was evaporated in vacuo and the solids were redissolved in the minimum amount of $\mathrm{CS}_{2}$ and charged to a silica gel column overnight. After silica gel hydrolysis, unreacted fullerene and product were separated by flash chromatography with hexane/CS $2: 1$, then with toluene. The obtained brown powder was dried in vacuo to yield $430 \mathrm{mg}$ of $\mathrm{FUL}=\mathrm{O}$ (39\% yield). $200 \mathrm{mg}$ $(0.253 \mathrm{mmol})$ of $\mathrm{FUL}=\mathrm{O}$ was dissolved in $20 \mathrm{~mL}$ of dry toluene. $0.8 \mathrm{~mL}(0.8 \mathrm{mmol})$ of a $1.0 \mathrm{M}$ solution of diisobutylaluminium hydride (DIBAL-H) in cyclohexane was added dropwise. The reaction mixture was stirred for $3 \mathrm{~h}$ at room temperature and then stirred with $40 \mathrm{~mL}$ of saturated sodium chloride for 2 h. Subsequently, the organic layer was separated and the aqueous layer was twice extracted with $50 \mathrm{~mL}$ of toluene. Combined organic phases were dried and the solvent evaporated. The dry residue was purified by flash chromatography 
(toluene) to yield $170 \mathrm{mg}(85 \%)$ of the black solid. ${ }^{1} \mathrm{H}$ and ${ }^{13} \mathrm{C}$ NMR data are discussed further herein.

\subsection{Synthesis of fullerene-linked poly(3,4-dimethoxystyrene) (PDMS-FULP or PDMS-FUL)}

A round-bottomed flask, equipped with a magnetic follower, was charged with $500 \mathrm{mg}$ of PDMS with molar mass ca. $5,000 \mathrm{~g} / \mathrm{mol}$ (ca. $0.100 \mathrm{mmol}$ ) and $0.140 \mathrm{mmol}$ of fullerenol precursor. The contents of the flask were dissolved in dry toluene and dichloromethane $(4: 1)$ upon sonication. DCC (29 mg, $0.140 \mathrm{mmol}$ ) and DMAP (17 mg, $0.140 \mathrm{mmol}$ ) were added to the solution. The flask was sealed, purged with nitrogen through a needle and then its contents stirred for 38 hours at $60^{\circ} \mathrm{C}$. Once the resulting mixture was cooled to room temperature, it was filtered and the solvent was subsequently evaporated. The solids were redissolved in cold THF (5 mL), filtered, washed with cold THF and concentrated before precipitating into cold hexane $(200 \mathrm{~mL})$. Finally, the precipitate was collected by filtration, washed with hexane several times and dried in vacuo to obtain a brown powder (577 mg, yield $96 \%$ ). The same procedure was employed to obtain PDMS-FULP.

\subsection{Characterisation}

${ }^{1} \mathrm{H}$ and ${ }^{13} \mathrm{C}$ NMR spectra were recorded using a Bruker NMR spectrometer $(300 \mathrm{MHz})$. All chemical shifts are reported in ppm (ס) and referenced to the chemical shifts of the residual solvent resonances. Fourier transform infrared (FTIR) spectra were obtained using attenuated total reflectance (ATR) on a Thermo Nicolet 380 FTIR spectrophotometer over the range $4000-500 \mathrm{~cm}^{-1}$ for 32 scans with a resolution of $4 \mathrm{~cm}^{-1}$. Number-average molecular mass $\left(M_{\mathrm{n}}\right)$ and dispersity $\left(M_{\mathrm{w}} / M_{\mathrm{n}}, \bigoplus\right)$ were measured using gel permeation chromatography (GPC) (flow rate $1 \mathrm{~mL} / \mathrm{min}$ ) with a refractive index $(\mathrm{RI})$ detector through three $\mathrm{PL}$ gel $5 \mathrm{~mm} 300 \times 7.5$ $\mathrm{mm}$ mixed-C columns using a degassed THF eluent system containing $2 \%(\mathrm{v} / \mathrm{v})$ TEA. The system, operating at $40^{\circ} \mathrm{C}$, was calibrated with narrow polystyrene standards $\left(M_{p}\right.$ range $=162$ to $6035000 \mathrm{~g} / \mathrm{mol}$ ). All data were analysed using PL Cirrus software (version 2.0) supplied by Agilent Technologies (previously Polymer Laboratories). $M_{\mathrm{n}}$ was also evaluated using ${ }^{1} \mathrm{H}$ 
NMR spectroscopy (via end group analysis). Thermogravimetric analysis (TGA) was performed on Pyris 1 TGA instrument under nitrogen atmosphere with a heating rate of 10 ${ }^{\circ} \mathrm{C} / \mathrm{min}$. For MALDI analysis, Bruker Ultraflextreme TOF instrument equipped with a nitrogen laser (337 nm) laser was operated in linear positive mode ion mode, between m/z 3000-10000. Dithranol was used as matrix and sodium iodide as a mild cationising agent.

\section{RESULTS AND DISCUSSION}

The synthesis of fullerene-functionalised polymer involved three steps (Scheme 1): (i) RAFT polymerisation; (ii) synthesis of hydroxy-functionalised fullerene (fullerenol) via either route 1 or route 2 and (iii) Steglich esterification to couple the polymer to fullerenol. As a first step, RAFT polymerisation of 3,4-dimethoxystyrene (DMS) was employed to produce the polymer, PDMS, as previously demonstrated by our group. ${ }^{32}$ PDMS, chosen as an exemplar in this study, has found multiple uses as a precursor to its dopamine-like hydroxylated analogue or due to its own intrinsic metal-binding properties. ${ }^{32,33}$ However, it is important to note that RAFT is not exclusively limited to PDMS and is capable of polymerising a wide range of versatile monomers, from sulfonated styrenics, chlorinated divinyl moieties and elaborate quinoline derivatives to a whole host of stimuli-responsive monomers. ${ }^{23,34-39}$ Moreover, RAFT permits the fabrication of well-defined polymers with high monomer conversion and low molar mass dispersity over a range of molecular masses. ${ }^{34,40-43}$ The chain transfer agent (CTA), the key reagent in the RAFT polymerisation, provides chain end functionality, allowing, in the case herein, PDMS to be furnished with a terminal carboxylic acid group [step (i)].

The second step of the procedure was the fabrication of a hydroxy-functionalised fullerene derivative via one of two possible routes, denoted as fullerenol FULP-OH or FUL-OH (for route 1 or route 2 , respectively). To identify the simplest, most appropriate and straightforward method, two well-reported routes were tested, based on two different types of cycloaddition; the Prato reaction (route 1) and Diels-Alder cycloaddition (route 2). 
The third step consisted of coupling the products from steps (i) and (ii) (obtained by either routes 1 or 2) using DCC as an acid-activating reagent and DMAP as a base. To achieve addition of the fullerene moiety to every polymer chain in step (iii), excess fullerenol (FULP$\mathrm{OH} / \mathrm{FUL}-\mathrm{OH}$ ) was used.

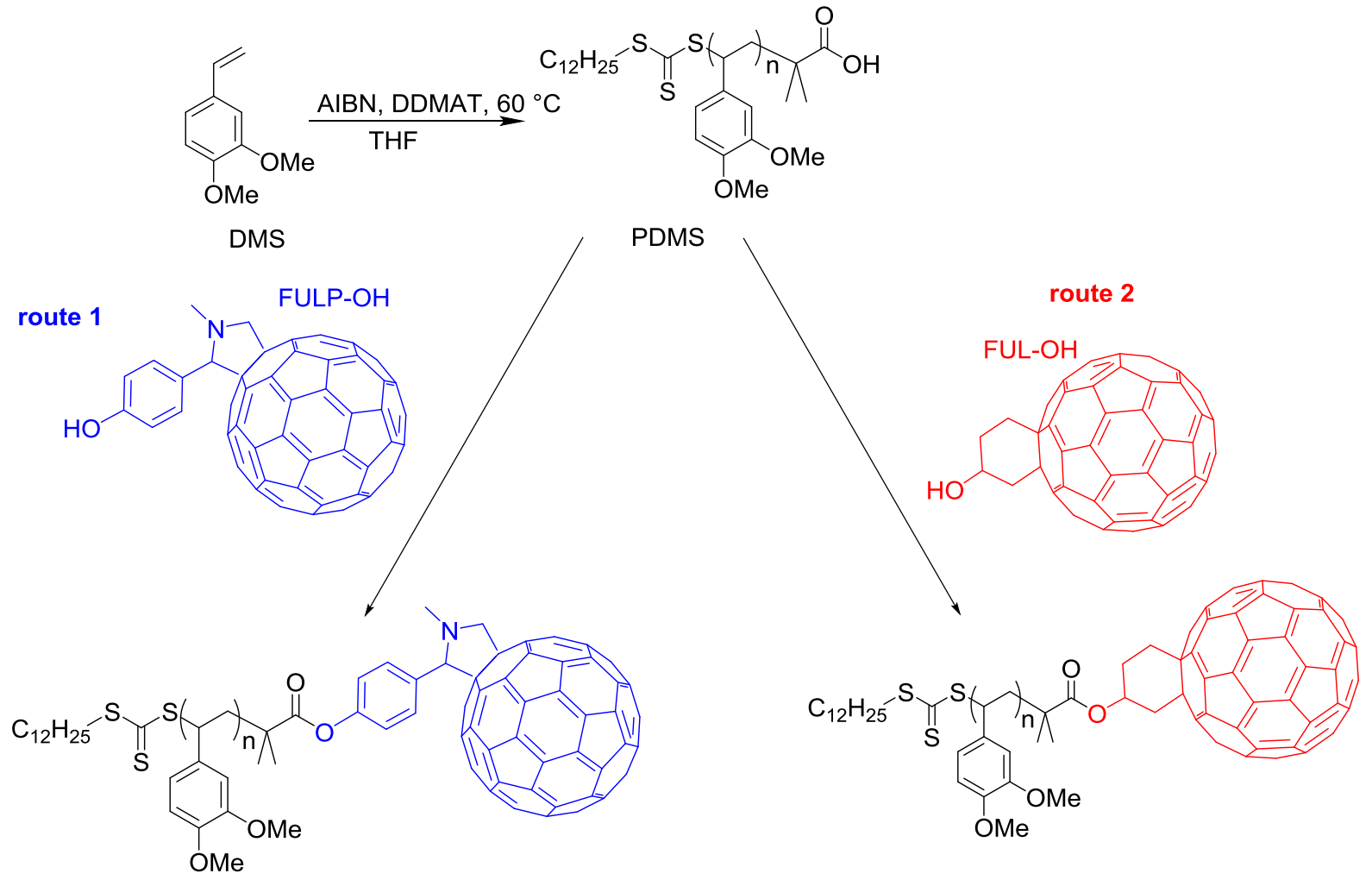

Route 1 precursor:
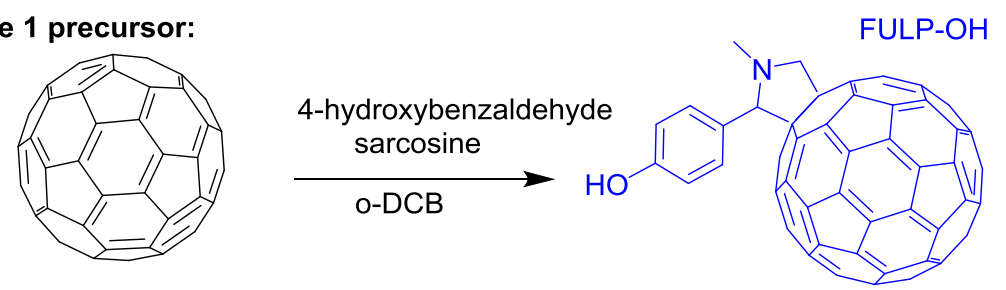

Route 2 precursor:
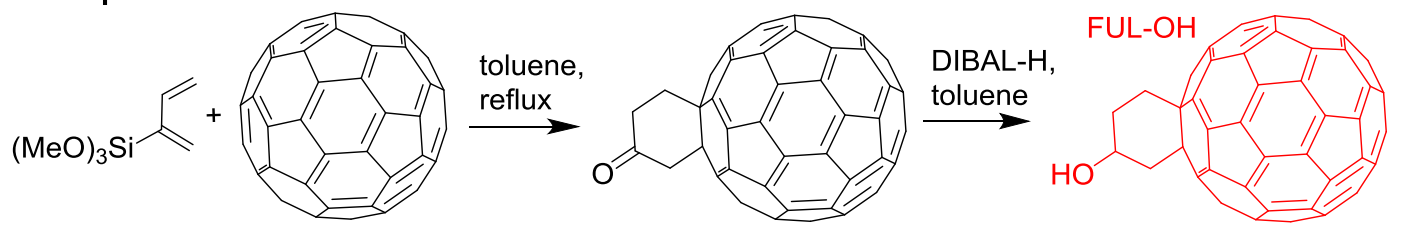

Scheme 1. Synthesis of fullerene-coupled PDMS via two routes. Route 1 involves a Prato reaction to generate fullerenol (FULP-OH) and yields PDMS-FULP, whereas Route 2 
proceeds through a Diels-Alder fullerene derivative (FUL-OH) to yield PDMS-FUL. The scheme for generation of route 1 and route 2 precursors is also presented.

\section{Route 1}

The Prato reaction was carried out according to previous reports, using amino acid sarcosine and hydroxyl-functionalised benzaldehyde to generate azomethine in situ. ${ }^{44,45}$ Furthermore, 4hydroxybenzaldehyde, which provided the alcohol moiety for the final product, did not require protection, thus the reaction proceeded in one simple step. The product, FULP-OH, was then coupled to PDMS polymer via Steglich esterification to yield PDMS-FULP. Success of the coupling reaction was confirmed by ${ }^{1} \mathrm{H}$ NMR spectroscopy (Figure 1) and gel permeation chromatography (GPC) (Figure 2). The ${ }^{1} \mathrm{H}$ NMR spectrum shows the characteristic peaks for methylene protons of the pyrrolidine cycle of the Prato derivative (labelled as $a, b$ and $c$ in Figure 1) and $\mathrm{N}$-methyl protons (labelled as $f$ ) in the coupling product, although $\mathrm{H}_{a}$ is partially obscured by the methoxy proton peaks originating from the PDMS repeat unit. The corresponding benzene ring proton peaks ( $e$ and $d$ ) are no longer discernible in the spectrum of the coupled product since the concentration of these protons decreases whilst their chemical environment changes. Accordingly, the peaks appear to have shifted and/or broadened so that they have become obscured. Notably, the peaks $a$ and $b$ in the FULP product have significantly different chemical shifts due to their corresponding cis and trans positions. The ${ }^{13} \mathrm{C}$ NMR spectrum of PDMS-FULP (Figure S2, ESI) is essentially a combination of the ${ }^{13} \mathrm{C}$ spectra of PDMS and precursor FULP-OH, with a broad pattern for quarternary carbon peaks of the non-symmetrical fullerene, again corroborating the success of the coupling reaction. 
(a)

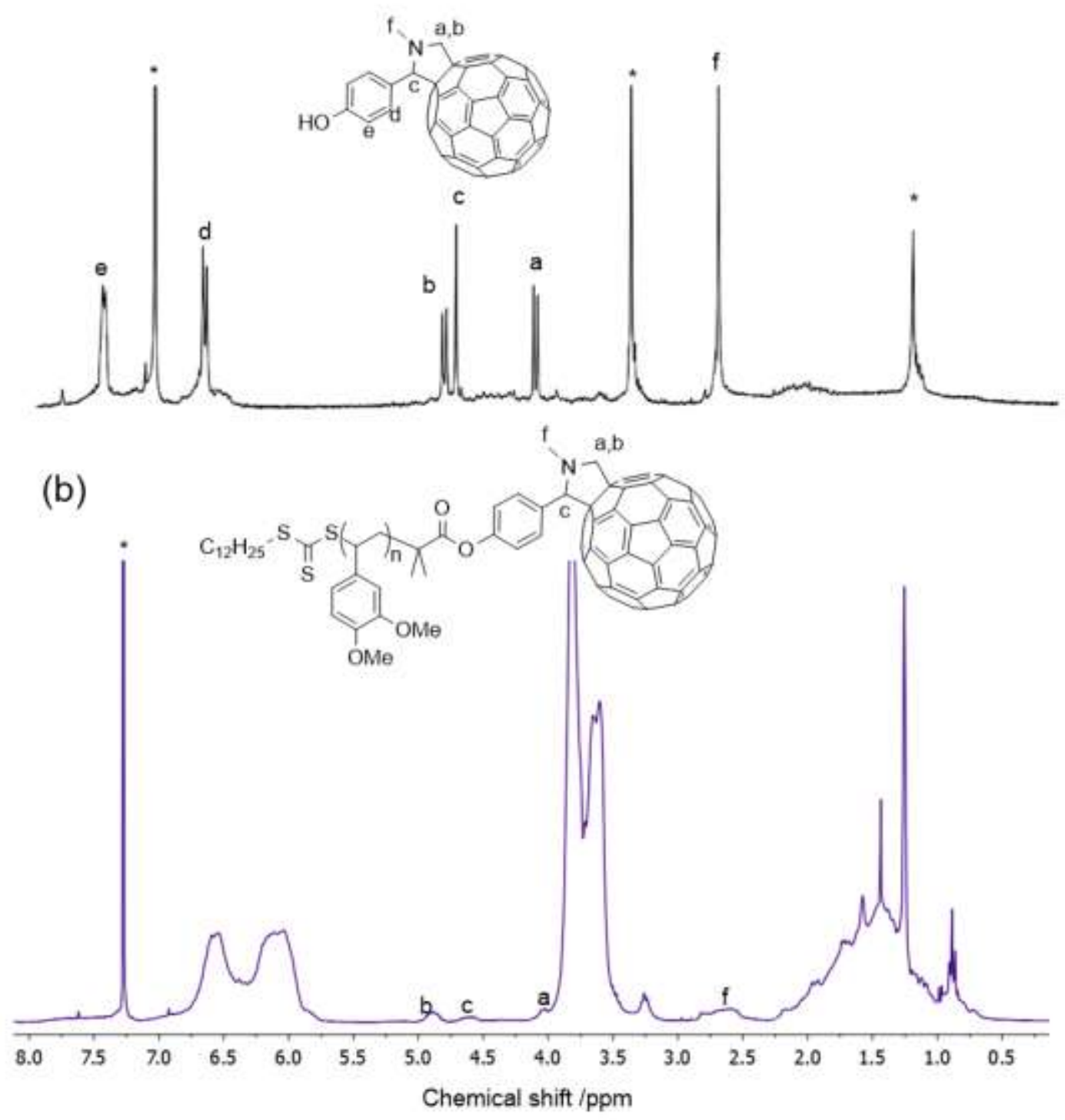

Figure 1. ${ }^{1} \mathrm{H}$ NMR spectra of the FULP starting material (a) and PDMS-FULP product (b). Residual solvent peaks are labelled with asterisks. For simplicity, only the structure of the monoadduct is shown in (b).

The GPC traces in Figure 2 show a complex peak pattern which does not agree with the expected monotonic shift in retention time. Firstly, the initial GPC peak has been shifted towards much higher molar mass, as expected from addition of a C60 molecule $(\sim 1,280 \mathrm{Da}) \cdot{ }^{24}$ Secondly, the peak pattern of the GPC trace is characteristic of two distinct polymer products with a two-fold difference in molecular mass, as obtained from peak deconvolution. This rather 
unexpected complex GPC pattern is attributed to both mono- and bis- addition of PDMS chains onto a single fullerene precursor and is clearly a result of the double functionalisation of the fullerene in the Prato reaction. The possibility of multiple additions in the Prato reaction is low, but not excluded, as demonstrated elsewhere,${ }^{46}$ thus the bis-adduct is an unavoidable reaction product that can be difficult to discern by NMR spectroscopy, and could only be observed during characterisation of the final PDMS-FULP coupled material. The ratio of the bis- to monoadduct is approximately 0.8 to 1 as estimated from GPC peak deconvolution. Additionally, no residual unbound PDMS was observed, indicating that conversion of PDMS into either of PDMS-FULP adducts was quantitative. A slight aggregation-induced shoulder is observed at early retention time for (PDMS) ${ }_{2}$-FULP peak.
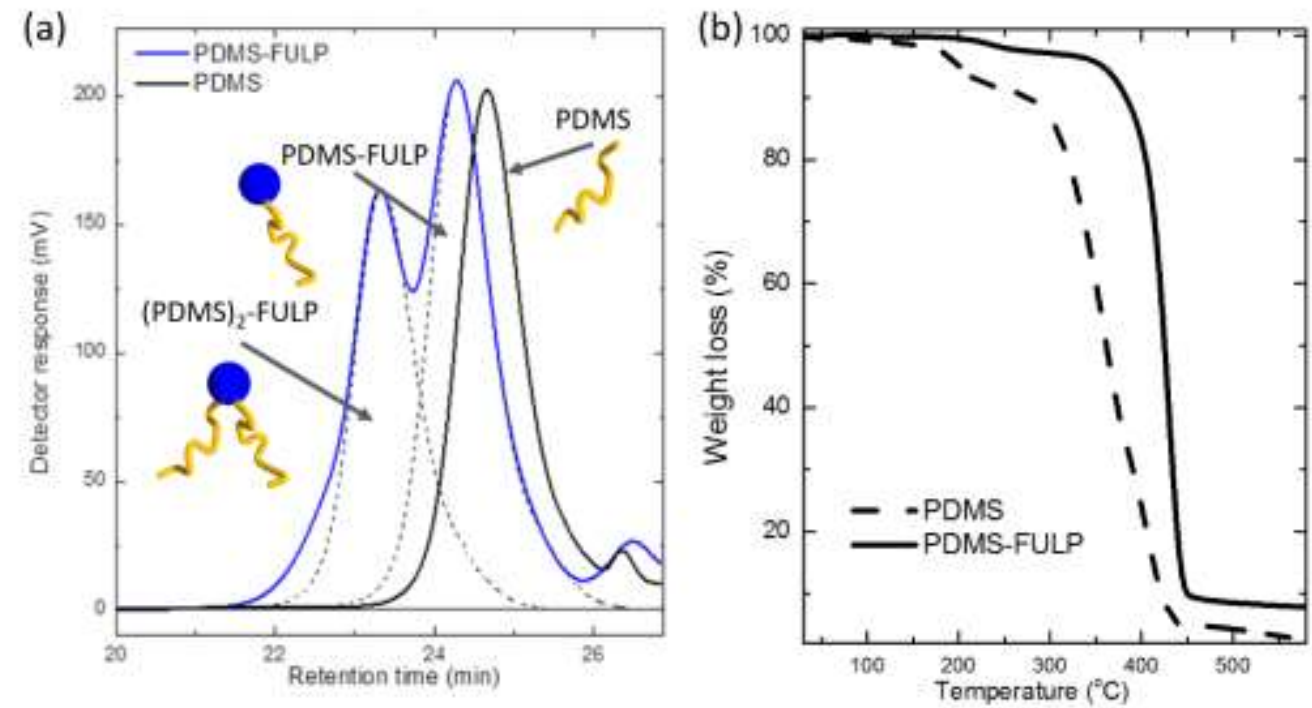

Figure 2. (a) GPC traces of the starting material PDMS and the resulting PDMS-FULP product. Deconvolution traces are also shown as dotted lines, representing the (PDMS $)_{2^{-}}$ FULP bisadduct and PDMS-FULP monoadduct. (b) TGA curves of PDMS (dashed line) and PDMS-FULP (solid line) product.

Thermogravimetric analysis of PDMS and PDMS-FULP (Figure 2b) was performed to analyse the char content, indicative of the fullerene content. $8,47,48$ TGA has shown that precursor PDMS decomposed with 0.5\% residual char, whereas PDMS-FULP demonstrated 
a higher char yield of $8.9 \%$, attributed to the residual $\mathrm{C60}$, however, smaller than expected for mono-addition of a single polymer chain onto one fullerene molecule $(12.6 \%+0.5 \%=13.1 \%)$, thus in agreement with observations from GPC that di-adducts were also generated during the Prato route (route 1). Importantly, the onset of PDMS-FULP major decomposition is shifted to later time $\left(405^{\circ} \mathrm{C}\right)$ as compared to pristine PDMS $\left(323^{\circ} \mathrm{C}\right)$. As previously demonstrated, the carboxylic acid group of the PDMS is labile at high temperatures and the esterification of this bond leads to a decrease in this lability. ${ }^{49}$ Additionally, fullerene possesses a high radical scavenging capability and appears to retard degradation associated with radical formation within the polymer. ${ }^{47}$

The results of elemental analysis also confirm the presence of di-adducts in the final product, where the experimental carbon content is lower than theoretical and the content of sulfur and oxygen is higher, corroborating that more than one chain of PDMS was added onto a fullerene molecule (see ESI).

\section{Route 2}

Route 2 was based on well-reported Diels-Alder cycloaddition reaction which proceeded in two steps. ${ }^{12,24,50}$ First, the 1,2-(4'-oxocyclohexano)fullerene was made (Scheme 1, Route 2) and then reduced by a relatively mild DIBAL-H agent to give a stable secondary alcohol (FUL$\mathrm{OH})$. The resulting fullerenol was coupled to PDMS according to a general esterification procedure, described in the Experimental Section. Figure 3 shows the ${ }^{1} \mathrm{H}$ NMR spectra of the PDMS and FUL-OH precursor materials alongside the PDMS-FUL product. A cosolvent system of deuterated chloroform and acetone was required for the fullerene precursor, due to its insufficient solubility in a single common solvent. ${ }^{51}$ The ${ }^{1} \mathrm{H}$ NMR spectrum of the coupled polymer shows two new peaks appearing at $3.5 \mathrm{ppm}$ and $4.0-4.1 \mathrm{ppm}$, attributed to the methylene protons on the fullerene moiety (Fig. 3a). These peaks are difficult to assign precisely as the methylene peaks of the fullerene precursor between 3.5 and $3.8 \mathrm{ppm}$ are 
overlapping and cannot be resolved. Additionally, these peaks cannot be accurately integrated because they overlap with the methoxy protons of the PDMS moiety at 3.5 - $4.0 \mathrm{ppm}$ (labelled with a triangle in Figure 3). Importantly, the presence and shifting of peaks in the FUL-OH cyclohexyl ring provide strong evidence that PDMS and fullerene are covalently bound to one another. The multiplet peak at 5.2 - $5.3 \mathrm{ppm}$ is attributed to the proton attached to the secondary carbon of FUL-OH $\left(\mathrm{H}_{\mathrm{a}}\right)$, which upon esterification is significantly shifted downfield, where it is obscured by the peaks of the aromatic protons (expected at $6.1-6.6 \mathrm{ppm}$ ). ${ }^{24,50}$ Importantly, the coupled product shows a more defined peak around $3.2 \mathrm{ppm}$ from one of the methylene protons attached to the carbon adjacent to the secondary carbon (labelled $b$ in Fig. $3 a, c){ }^{24}$. The integration of this peak to the peak of the CTA methylene protons is 1 to 2 , suggesting pure PDMS-FUL material. All remaining peaks arising from the cyclohexyl methylene protons of the fullerene derivative cannot be established as they are completely obscured by the methoxy peaks from PDMS at 3.5-4.0 ppm. Peak $c$, which is observed in the spectrum of FULP-OH, is only slightly visible in the FULP-OH spectrum. This is associated with the shift of the peaks in the coupled material, accompanied by the solvent variations, thus the peak is obscured by the protons of the PDMS backbone. As further evidence, ${ }^{13} \mathrm{C}$ NMR spectroscopy was used to characterise the final product (Figure S1, ESI), where the spectrum is a close resemblance of a combination of the spectra of the two starting materials, clearly showing contributions from both PDMS (most notably at approximately 55, 110 and 120 ppm) and fullerene (135 - $139 \mathrm{ppm})$. 

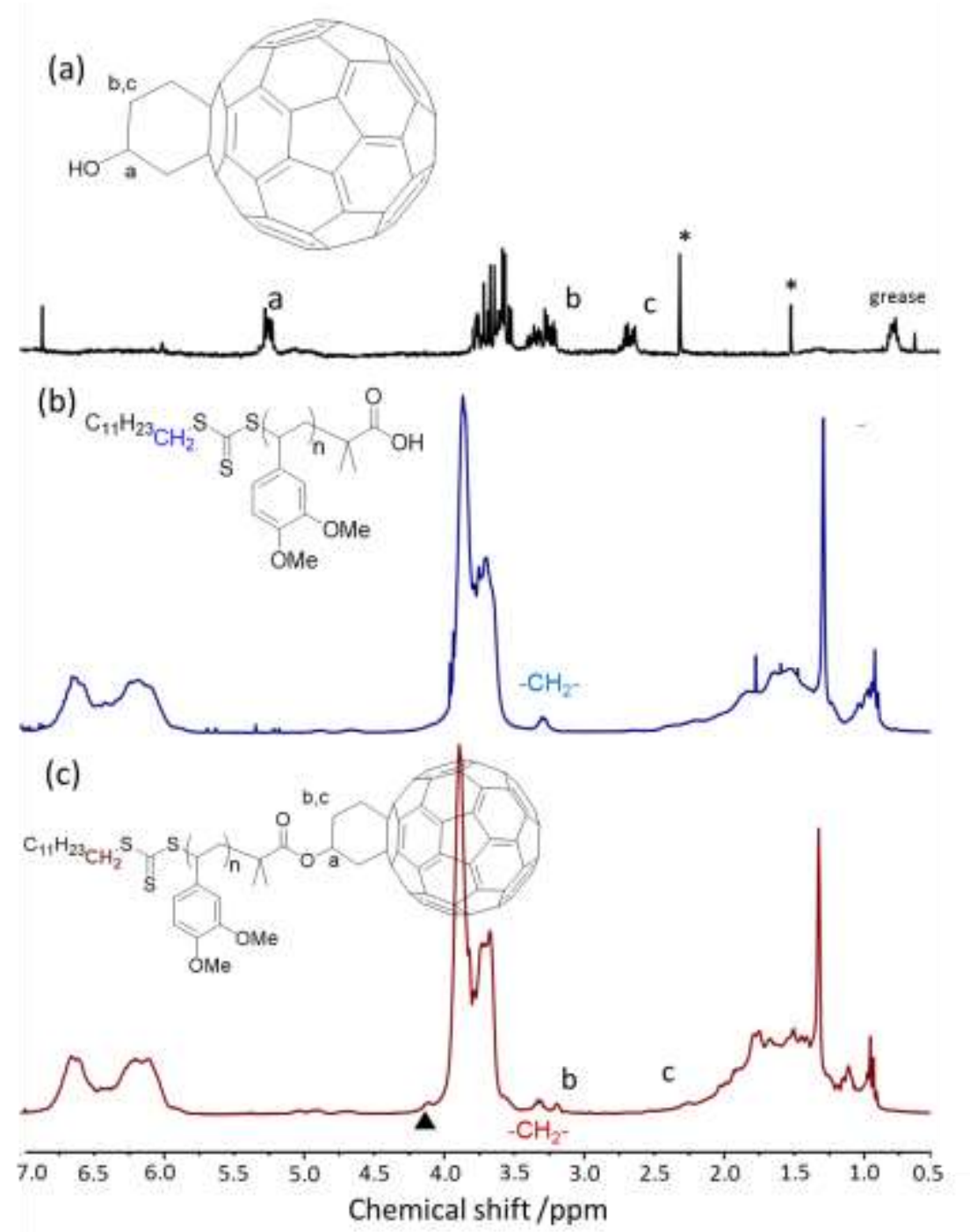

Figure 3. ${ }^{1} \mathrm{H}$ NMR spectra of (a) fullerenol precursor FUL-OH $\left(\mathrm{d}_{6}\right.$-acetone:CDCl 3 ), (b) PDMS (in $\mathrm{CDCl}_{3}$ ) and (c) resulting PDMS-FUL (in $\mathrm{CDCl}_{3}$ ). Unresolved peaks originating from the methylene protons of FUL-OH in PDMS-FUL product are labelled with a triangle. Residual solvent peaks are labelled with asterisks.

The GPC traces of PDMS and PDMS-FUL are given in Fig. 4a (FUL-OH could not be analysed due to its insolubility in common solvents). As expected, a small shift to higher molecular mass was observed for PDMS-FUL (from $5,000 \mathrm{~g} / \mathrm{mol}$ to $5,400 \mathrm{~g} / \mathrm{mol}$ ), indicating the covalent attachment of a single fullerene molecule to the end of the PDMS chains. The shift in molar masses is slightly lower than expected for a fullerene addition ( 775 g/mol). This is a general property of all fullerene-functionalised polymers and is associated with the $\pi-\pi$ interactions of 
the fullerenes with the column packing material, thus, increasing the retention time. ${ }^{52,53}$ Moreover, the monotonic shift of the main peak suggests that there is negligible free PDMS impurity in the final product. The high molecular mass shoulder observed is attributed to aggregation of the PDMS-FUL fullerene moieties in THF, as reported elsewhere for fullerenefunctionalised polymers. ${ }^{24,50}$ No bis-adduct peaks were found for PDMS-FUL, in contrast to PDMS-FULP (synthesised via route 1), indicating that the FUL-OH precursor was a monoadduct with negligible traces of multiple adducts, if any.

The MALDI-TOF spectra of the polymers are shown in Figure 4b. Unfortunately, PDMS and PDMS-FUL polymers are very difficult to ionise and the quality of the spectral resolution was limited since the measurements were performed in linear mode. Thus, the precise information about the end groups and isotope distribution is not available. Nevertheless, it is clear to see that the PDMS spectrum is a Gaussian distribution of peaks with a maximum at $~ 4542 \mathrm{Da}$. This $\mathrm{m} / \mathrm{z}$ value corresponds to fragmentation of the CTA trithioester group during ionisation, in line with observations made before by various groups ${ }^{54-56}$ Interestingly, for dithioesters and trithioesters fragmentation occurs even when using mild ionising agents such as sodium iodide, as compared to silver or copper salts. ${ }^{56}$ Strube et al. suggested that lability of the CTA dithioester group to ionisation is associated with its absorbance maximum being very close to the laser frequency $(337 \mathrm{~nm})$, which is also valid for trithioesters (310 $\mathrm{nm}$ absorbance maximum). ${ }^{57}$ The fragmentation of PDMS polymer is illustrated in Scheme 2. Notably, there are no observable initiator-terminated peaks in MALDI spectrum, ${ }^{32}$ which is in agreement with our previous report. ${ }^{54}$ On the other hand, the PDMS-FUL spectrum does not have a perfectly Gaussian distribution. This is associated with the fact that smaller coupled molecules are easier to ionise than higher molecular weight molecules. Although the spectrum of PDMS-FUL is rather noisy, it clearly shows that the peaks correspond to PDMS plus $774 \mathrm{Da}$ (which is the mass of functionalised fullerene). The same fragmentation peaks are observed, as for PDMS, rather than intact polymeric product, however, the ester bond remained stable to ionisation, in 
agreement with Ladaviere et al. ${ }^{54}$ The absence of other major peaks in PDMS-FUL spectrum shows that the product is free from the starting material.
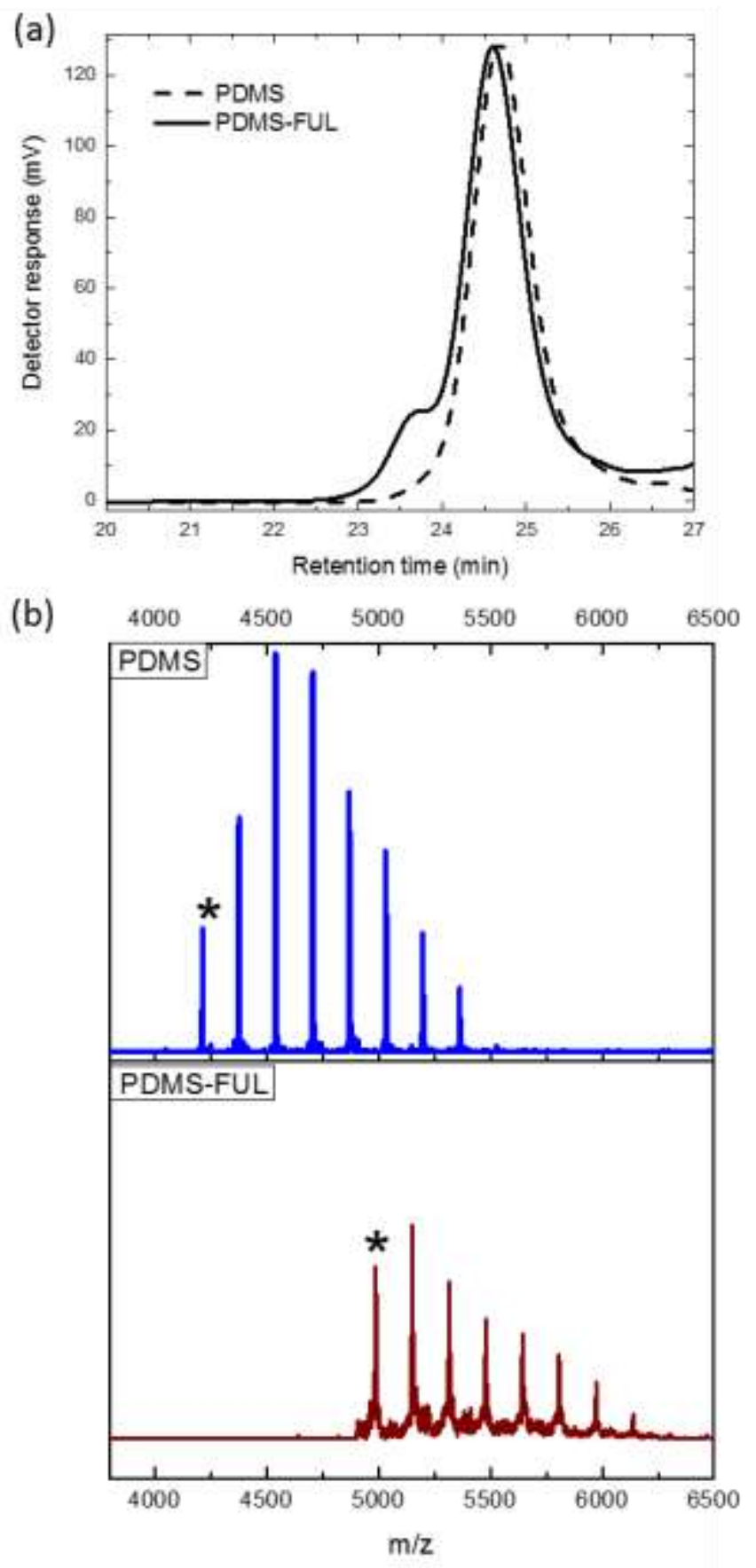

Figure 4. (a) GPC traces and (b) normalised intensity MALDI-TOF spectra of PDMS (blue) and PDMS-FUL (red). The peaks labelled with asterisks represent the beginning of the series at $D_{p}=25$. 


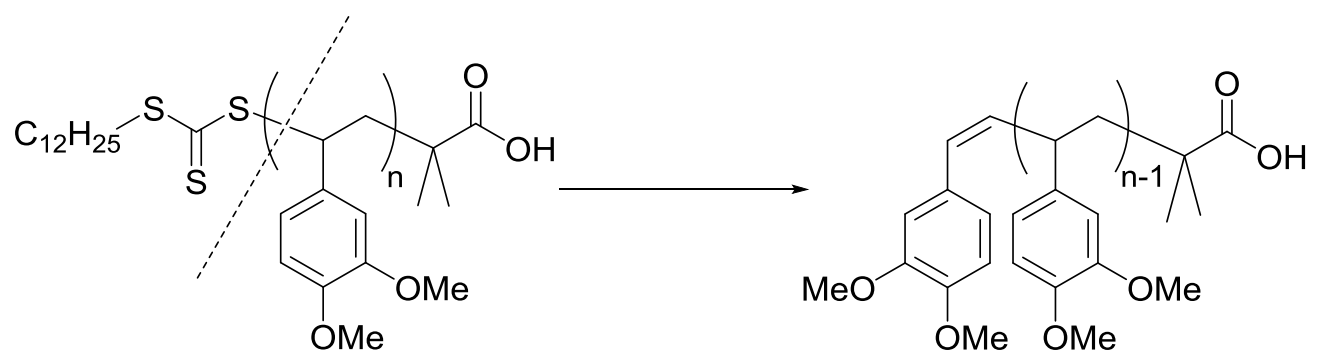

Scheme 2. Fragmentation of the end group of the CTA during ionisation of MALDI-TOF.

TGA of PDMS and PDMS-FUL (Figure 5a) was performed to confirm the absence of bisadducts. PDMS-FUL demonstrated higher char yield of $13.9 \%$ than PDMS $(0.5 \%)$, only marginally higher than that expected from addition of one fullerene per molecule $(12.6 \%+$ $0.5 \%=13.1 \%$ ) and within the instrumental error. The decomposition profile of the PDMS-FUL polymer changes, with major decomposition occurring later than for pristine PDMS $\left(363^{\circ} \mathrm{C}\right.$ and $323^{\circ} \mathrm{C}$, respectively). Same effect was observed for PDMS-FULP product in route 1 , suggesting that the major decomposition is mediated by the carboxylic acid end group which is stabilised by the fullerene attached to it in PDMS-FUL and PDMS-FULP.
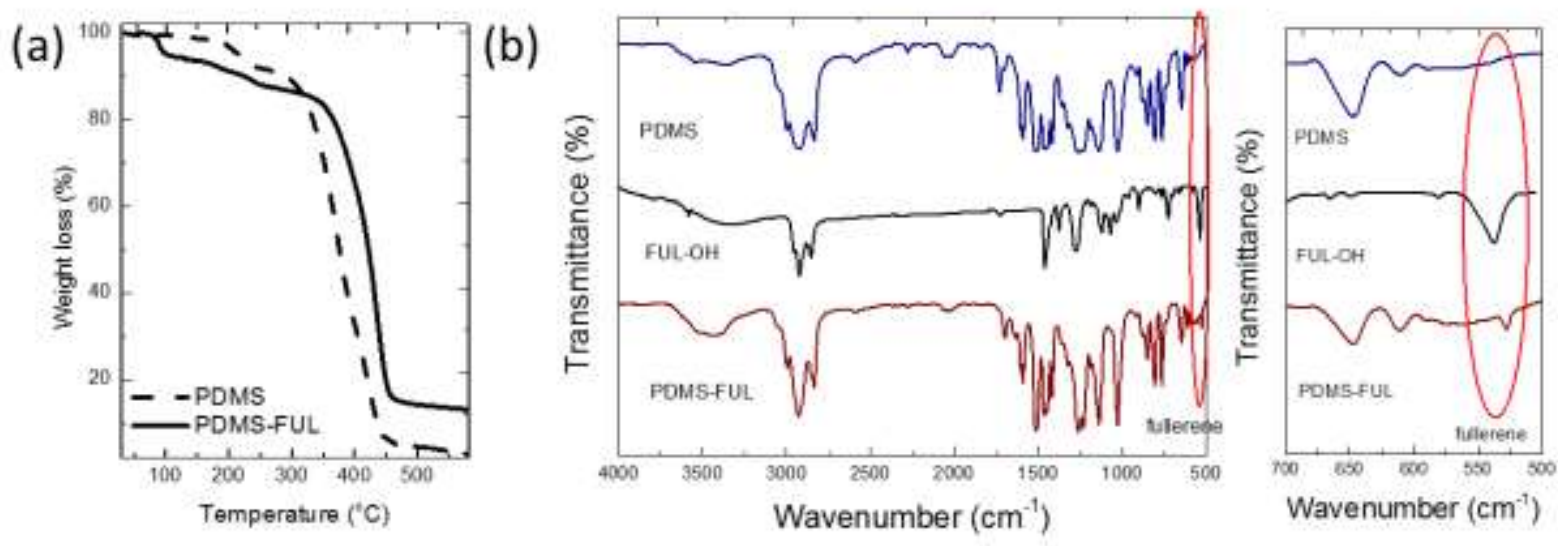

Figure 5. TGA curves (a) of PDMS and PDMS-FUL polymers. (b) FTIR and spectra of PDMS (blue), FUL-OH (black) and PDMS-FUL (red). 
Figure $5 \mathrm{~b}$ shows the FTIR spectra of the PDMS and fullerenol starting materials alongside the PDMS-FUL product. The band at $527 \mathrm{~cm}^{-1}$ is indicative of the fullerene being present in our macromolecule. ${ }^{58}$ The characteristic carbonyl band of the carboxylic acid chain end on PDMS shifted from $1730 \mathrm{~cm}^{-1}$ (typical for a non-hydrogen bonded carboxylic acid group ${ }^{59}$ ) to $1700 \mathrm{~cm}^{-1}$ in agreement with previous reports for fullerene-coupled materials ${ }^{60}$, although lower than expected for a typical carbonyl ester band. The absence of the carboxylic acid carbonyl group in the coupled PDMS-FUL material provides further evidence that there is no unreacted polymer remaining. It should be noted that the $\mathrm{O}-\mathrm{H}$ stretch evolution observed at $3500 \mathrm{~cm}^{-1}$ is associated with residual water that is difficult to completely eliminate.

Although providing a slightly more rapid procedure for fabricating the fullerenol precursor, route 1 has the major disadvantage of resulting in multiple adducts that requires laborious separation and purification stages to remove the bis-adducts. On the other hand, multiple adducts in the Prato reaction could be useful for the fabrication of star-shaped fullerene derivatives, for example, provided that a level of control can be attained over the precise number of additions. ${ }^{61}$ At the same time, route 2 had one more step in the FUL-OH synthesis, however, yielded only mono-adducts which were then readily coupled to the PDMS chain end and thus resulted in well-defined fullerene-functionalised macromolecules. To demonstrate the scope of this procedure to produce polymeric constructs of various sizes, PDMS-FUL polymers of two other molecular masses were fabricated: $10,000 \mathrm{~g} / \mathrm{mol}$ and 50,000 g/mol (Figure S4). Slight monotonic shifts in the GPC traces were observed for both products, confirming successful coupling. Interestingly, however, higher molecular mass PDMS appears to suppress the presence of fullerene aggregation in THF, suggesting that the tethered macromolecules have surfactant-like properties, with longer chains having the ability to cover more of the large, fullerene spherical surface area.

\section{CONCLUSIONS}

For the first time, this paper reports a facile and elegant way to couple individual chains of poly(3,4-dimethoxystyrene), via a chain-end carboxylic acid group furnished during the RAFT 
polymerisation process, to a single fullerene molecule. The polymer was coupled to a hydroxyfunctionalised fullerene using Steglich esterification. Two routes to produce the functionalised fullerene were explored; the Prato reaction (to give FULP-OH) and a Diels-Alder cycloaddition $(\mathrm{FUL}-\mathrm{OH})$. Although requiring one less synthetic step, the Prato reaction gave rise to a mixture of polymeric constructs [namely PDMS-FULP and (PDMS $)_{2}-\mathrm{FULP}$ ] that were difficult to separate, as observed by GPC. Diels-Alder cycloaddition (to produce FUL-OH) constituted a two-step procedure, but resulted in conversion of PDMS into a mono-addition product, PDMSFUL, with very high yield (96\%), where PDMS was selected as a model polymer for the synthetic approaches reported herein (owing to its previously reported metal-binding properties). Thus, the Diels-Alder route was found highly suitable for functionalisation of a diverse range of RAFT-generated polymers of different molecular masses, as demonstrated for 5,000, 10,000 and $50,000 \mathrm{~g} / \mathrm{mol}$ PDMS-FUL.

\section{Acknowledgements}

The research leading to these results has received funding from the European Union Seventh Framework Programme (FP7/ 2011 under grant agreement ESTABLIS no. 290022).

\section{References}

1 H. W. Kroto, J. R. Heath, S. C. O'Brien, R. F. Curl and R. E. Smalley, Nature, 1985, 318, 162-163.

2 C. Wang, Z. Tao, W. Yang and S. Fu, Macromol. Rapid Commun., 2001, 22, 98-103.

3 Y. Chen, R. Cai, L. Xiao, Z. Huang and Y. Chen, J. Mater. Sci., 1998, 3, 4633-4641.

4 J. Yang, L. Li and C. Wang, Macromolecules, 2003, 36, 6060-6065.

$5 \quad$ M. S. Bratcher, M. S. DeClue, A. Grunnet-Jepsen, D. Wright, B. R. Smith, W. E. Moerner and J. S. Siegel, J. Am. Chem. Soc., 1998, 120, 9680-9681.

6 Y. Wang, H. Benten, S. Ohara, D. Kawamura, H. Ohkita and S. Ito, ACS Appl. Mater. Interfaces, 2014, 6, 14108-14115. 
Absalon and H. Cramail, Macromolecules, 2009, 42, 3549-3558.

8 M. Heuken, H. Komber, T. Erdmann, V. Senkovskyy, A. Kiriy and B. Voit, Macromolecules, 2012, 45, 4101-4114.

9 P. D. Topham, A. J. Parnell and R. C. Hiorns, J. Polym. Sci. Part B-Polymer Phys., $2011,49,1131-1156$.

10 J. Yang, L. B. Alemany, J. Driver, J. D. Hartgerink and A. R. Barron, Chem. Eur. J., 2007, 13, 2530-2545.

11 C.-M. Lin, Recent Pat. Nanotechnol., 2012, 6, 105-113.

12 Y.-Z. An, C.-H. B. Chen, J. L. Anderson, D. S. Sigman, C. S. Foote and Y. Rubin, Tetrahedron, 1996, 52, 5179-5189.

13 C. Wang, Z.-X. Guo, S. Fu, W. Wu and D. Zhu, Prog. Polym. Sci., 2004, 29, 10791141.

14 J. Wootthikanokkhan, C. Thanachayanont and N. Seeponkai, J. Appl. Polym. Sci., 2010, 116, 433-440.

15 M. Hufnagel, M.-A. Muth, J. C. Brendel and M. Thelakkat, Macromolecules, 2014, 47, 2324-2332.

16 B. Gholamkhass, T. J. Peckham and S. Holdcroft, Polym. Chem., 2010, 1, 708.

17 C. J. Hawker, Macromolecules, 1994, 27, 4836-4837.

18 C. Weis, C. Friedrich, R. Mulhaupt and H. Frey, Macromolecules, 1996, 28, 403-405.

19 J. lehl, M. Urbani and J. Nierengarten, in Handbook of Carbon Nano Materials, 2010, pp. 1-32.

20 J. U. Lee, J. W. Jung, T. Emrick, T. P. Russell and W. H. Jo, J. Mater. Chem., 2010, 
$20,3287$.

21 J. W. Jung, J. W. Jo and W. H. Jo, Adv. Mater., 2011, 23, 1782-7.

22 D. J. Siegwart, J. K. Oh and K. Matyjaszewski, Prog. Polym. Sci., 2012, 37, 18-37.

23 G. Moad, M. Chen, M. Häussler, A. Postma, E. Rizzardo and S. H. Thang, Polym. Chem., 2011, 2, 492.

24 X.-H. Dong, W.-B. Zhang, Y. Li, M. Huang, S. Zhang, R. P. Quirk and S. Z. D. Cheng, Polym. Chem., 2012, 3, 124.

25 W.-B. Zhang, Y. Tu, R. Ranjan, R. M. Van Horn, S. Leng, J. Wang, M. J. Polce, C. Wesdemiotis, R. P. Quirk, G. R. Newkome and S. Z. D. Cheng, Macromolecules, $2008,41,515-517$.

26 S. Dai, P. Ravi, C. H. Tan and K. C. Tam, Langmuir, 2004, 20, 8569-8575.

27 P. Zhou, G. Chen, H. Hong, F. Du, Z. Li and F. Li, Macromolecules, 2000, 33, 19481954.

28 J. Y. Dong, E. Manias and T. C. Chung, Macromolecules, 2002, 35, 3439-3447.

29 D. E. Bergbreiter and H. N. Gray, J. Chem. Soc., Chem. Commun., 1993, 645-646.

30 K. E. Geckeler and A. Hirsch, J. Am. Chem. Soc., 1993, 115, 3850-3851.

31 Y. Ederlé and C. Mathis, Macromol. Rapid Commun., 1998, 19, 543-547.

32 A. Isakova, P. D. Topham and A. J. Sutherland, Macromolecules, 2014, 47, 25612568.

33 B. P. Lee, P. B. Messersmith, J. N. Israelachvili and J. H. Waite, Annu. Rev. Mater. Res. Vol 41, 2011, 41, 99-132.

34 G. Gody, T. Maschmeyer, P. B. Zetterlund and S. Perrier, Nat. Commun., 2013, 4, 2505. 
37 A. Isakova, O. Efremova, N. Pullan, L. Lüer and P. D. Topham, RSC Adv., 2016, 6, 6598-6606.

38 H. Erothu, J. Kolomanska, P. Johnston, S. Schumann, D. Deribew, D. T. W. Toolan, A. Gregori, C. Dagron-Lartigau, G. Portale, W. Bras, T. Arnold, A. Distler, R. C. Hiorns, P. Mokarian-Tabari, T. W. Collins, J. R. Howse and P. D. Topham, Macromolecules, 2015, 48, 2107-2117.

39 I. F. Domínguez, J. Kolomanska, P. Johnston, A. Rivaton and P. D. Topham, Polym. Int., 2014, n/a-n/a.

40 G. Moad, E. Rizzardo and S. H. Thang, Aust. J. Chem., 2005, 58, 379-410.

41 G. Moad, M. Chen, M. Haeussler, A. Postma, E. Rizzardo and S. H. Thang, Polym. Chem., 2011, 2, 492-519.

42 G. Moad, E. Rizzardo and S. H. Thang, Chem. Asian J., 2013, 8, 1634-1644.

43 R. N. Carmean, T. E. Becker, M. B. Sims and B. S. Sumerlin, Chem, 2017, 2, 93-101.

44 M. Maggini, G. Scorrano and M. Prato, J. Am. Chem. Soc., 1993, 115, 9798-9799.

45 K. H. Le Ho and S. Campidelli, Adv. Nat. Sci. Nanosci. Nanotechnol., 2014, 5, 25008.

46 A. Montellano López, A. Mateo-Alonso and M. Prato, J. Mater. Chem., 2011, 21, $1305-1318$.

47 R. Katiyar, D. S. Bag and I. Nigam, Thermochim. Acta, 2013, 557, 55-60.

48 T. Pradeep, K. K. Singh, A. P. B. Sinha, D. E. Morris, K. Holczer, S. Donovan, G. Gruner, J. D. Thompson, R. M. Fleming, A. R. Kortan, S. H. Glarum, A. V. Makhija, A. J. Muller, R. H. Eick, S. M. Zahurak, R. Tycko, G. Dabbagh and F. A. Thiel, J. Chem. 
Soc., Chem. Commun., 1992, 12, 1747-1748.

49 Y. Hou, J. Jiang, K. Li, Y. Zhang and J. Liu, J. Phys. Chem. B, 2014, 118, 1962-1967.

50 W.-B. Zhang, J. He, X. Dong, C.-L. Wang, H. Li, F. Teng, X. Li, C. Wesdemiotis, R. P. Quirk and S. Z. D. Cheng, Polymer (Guildf)., 2011, 52, 4221-4226.

51 P. S. Ganapathi, S. H. Friedman, G. L. Kenyon and Y. Rubin, J. Org. Chem., 1995, 60, 2954-2955.

52 A. K. and and K. Müllen*, 1999.

53 M. G. Nava, S. Setayesh, A. Rameau, P. Masson and J.-F. Nierengarten, New J. Chem., 2002, 26, 1584-1589.

54 C. Ladavière, P. Lacroix-Desmazes and F. Delolme, Macromolecules, 2008, 42, 7084.

55 C. Schilli, M. G. Lanzendörfer and A. H. E. Müller, Macromolecules, 2002, 35, 68196827.

56 O. I. Strube, L. Nothdurft, M. Drache and G. Schmidt-Naake, Macromol. Chem. Phys., 2011, 212, 574-582.

57 H. Willcock and R. K. O'Reilly, Polym. Chem., 2010, 1, 149-157.

58 H. Kuzmany, R. Winkler and T. Pichler, J. Physics-Condensed Matter, 1995, 7, 66016624.

59 D. H. Williams and I. Fleaming, Eds., Spectroscopic methods in organic chemistry, McGraw-Hill Education, 2007.

60 T. W. Chamberlain, A. Camenisch, N. R. Champness, G. A. D. Briggs, S. C. Benjamin, A. Ardavan and A. N. Khlobystov, J. Am. Chem. Soc., 2007, 129, 86098614. 
61 H. H. Ramanitra, S. A. Dowland, B. A. Bregadiolli, M. Salvador, H. Santos Silva, D. Bégué, C. F. O. Graeff, H. Peisert, T. Chassé, S. Rajoelson, A. Osvet, C. J. Brabec, H.-J. Egelhaaf, G. E. Morse, A. Distler and R. C. Hiorns, J. Mater. Chem. C, 2016, 4, 8121-8129. 